\title{
Pleural plaques: markers of asbestos exposure or independent risk factor for pleural mesothelioma? A case report of mesothelioma developed on a pleural plaque in a worker with occupational asbestos exposure
}

\author{
d'Hauw G, Sisinni AG, Romeo R, Paolucci V and Sartorelli P* \\ Department of Medical Biotechnology, Unit of Occupational Medicine, Azienda Ospedaliera Universitaria Senese, University of Siena, Siena, Italy
}

\begin{abstract}
Pleural plaques are considered as a markers of asbestos exposure and not as an independent risk factor for the development of asbestos-related neoplastic diseases. However isolated studies showed an association between pleural plaques and malignat pleural mesothelioma. A clinical case of a patient with an history of occupational asbestos exposure affected by asbestosis, pleural plaques and pleural mesothelioma is presented.
\end{abstract}

\section{Introduction}

Pleural plaques (PPs) represent the most common non malignant asbestos-related disease (ARDs). Currently PPs are widely referred as a marker of asbestos exposure and not as an independent risk factor for the development of asbestos-related neoplastic diseases, in particular with regard to lung cancer [1]. However, the association between PPs and pleural mesothelioma (PM) remains controversial, as isolated studies showed a relationship between PPs and PM [2].

A clinical case of a patient with a history of occupational asbestos exposure affected by asbestosis and PPs is presented. During the follow up managed by the Occupational Medicine of Siena a PM was diagnosed at a PP.

\section{Case presentation}

82-year-old male worker in follow-up since 1998 for asbestosis and PPs. The patient worked at a geothermal station from 1960 to 1994. His work consisted in maintenance and repair of the industrial plant that implicated insulation removal before repairing and insulation of pipelines with asbestos. First hospitalization occurred in 1998 with the diagnosis of asbestosis and PPs. The mineralogical analysis of bronchoalveolar lavage fluid (BALF) with transmission electron microscopy (TEM) demonstrated an asbestos fibres concentration of $622 \mathrm{ff} / \mathrm{mL}$ BALF and $466 \mathrm{ff} / \mathrm{mL}$ BALF for amphibole and chrysotile respectively. A previous chest high-resolution computed tomography (HRCT) in 2013 showed a slight progression of the well known PPs. In March 2017, as the patient complained chest pain, dry cough and acute dyspnoea, another chest HRCT was performed demonstrating pleural effusion and wide thickening of the visceral pleura in which was possible appreciate areas of thickening. The histological examination carried out on pleural biopsies concluded for pattern consistent with desmoplastic mesothelioma on sclero-hyalin plaques. The case was discussed at the Multidisciplinary Oncology Group to evaluate the possible radiotherapy treatment.

\section{Discussion}

Although some studies on cohorts of asbestos exposed workers have shown correlation between PPs and PM [3], it is likely that this was the result of several factors including intensity and duration of exposure. Therefore PPs per se do not seem an independent risk factor for the development of neoplastic pleuropulmonary diseases [1]. Despite this, scientific literature is poor. Research has been focused on the relationship between PPs and lung cancer, while there is a lack of studies on the possible relationship between PPs and the development of PM. Actually the differentiation of PPs from early stages of PM is difficult, due to several overlapping radiological features [4,5]. Kato et al. [6] studied differences in the level and localization of pleural irregularities in early PM and benign asbestos pleural effusion using CT. They demonstrated that the level and localization of pleural irregularities significantly differed between patients with benign asbestos pleural effusion and PM. In this sense fluorodeoxyglucose PET/CT has been shown to be a useful diagnostic tool for differentiate PM from benign PPs [7]. Probably these difficulties have affected research in the specific field. In the reported case the intensity of exposure was demonstrated by the presence of asbestosis and the fibers concentration in BALF. This represents in itself a powerful risk factor for PM.

\section{Conclusion}

The widespread belief that there is no relationship between PPs and $\mathrm{PM}$ is not supported by a vast literature as it happens for the absence

Correspondence to: Pietro Sartorelli, Department of Medical Biotechnology, Unit of Occupational Medicine, Azienda Ospedaliera Universitaria Senese, University of Siena, Siena, Italy, Tel: 0577-235510; E-mail: pietro.sartorelli@ unisi.it

Key words: asbestos, asbestos exposure, asbestos-related diseases, pleural plaques, pleural mesothelioma

Received: June 23, 2017; Accepted: July 10, 2017; Published: July 13, 2017 
d'Hauw G (2017) Pleural plaques: markers of asbestos exposure or independent risk factor for pleural mesothelioma? A case report of mesothelioma developed on a pleural plaque in a worker with occupational asbestos exposure

of association between PPs and lung cancer. In the patient described it is not possible to determine whether the localization of the PM at a $\mathrm{PP}$ is incidental or not and whether the PM can be attributed only to intense exposure or even to the presence of PPs. Similar cases may not be uncommon even if not reported in the literature.

\section{References}

1. Maxim LD, Niebo R, Utell MJ (2015) Are pleural plaques an appropriate endpoint for risk analyses? Inhal Toxicol 27: 321-334. [Crossref]

2. Lewinsohn HC (1974) Early malignant changes in pleural plaques due to asbestos exposure: a case report. $\mathrm{Br} J$ Dis Chest 68: 121-127.
3. Pairon JC, Laurent F, Rinaldo M, Clin B, Andujar P, et al. (2013) Pleural plaques and the risk of pleural mesothelioma. J Natl Cancer Inst 105: 293-301. [Crossref]

4. Metintas M, Ucgun I, Elbek O, Erginel S, Metintas S, et al. (2002) Computed tomography features inmalignant pleural mesothelioma and other commonly seen pleural diseases. Eur J Radiol 41: 1-9. [Crossref]

5. Ng CS, Munden RF, Libshitz HI (1999) Malignant pleural mesothelioma: thespectrum of manifestations on CT in 70 cases. Clin Radiol 54: 415-421. [Crossref]

6. Kato K, Gemba K, Fujimoto N, Aoe K, Takeshima Y, et al. (2016) Pleural irregularities and mediastinal pleural involvement in earlystages of malignant pleural mesothelioma and benign asbestospleural effusion. Eur J Radiol 85: 1594-1600. [Crossref]

7. Prazakova S, Thomas PS, Sandrini A, Yates DH (2014) Asbestos and the lung in the 21st century: an update. Clin Respir J 8: 1-10. [Crossref]

Copyright: $(02017$ d'Hauw G. This is an open-access article distributed under the terms of the Creative Commons Attribution License, which permits unrestricted use, distribution, and reproduction in any medium, provided the original author and source are credited. 